\title{
Affine stratifications from finite misère quotients
}

\author{
Ezra Miller
}

Received: 28 July 2011 / Accepted: 18 February 2012 / Published online: 17 March 2012

(C) Springer Science+Business Media, LLC 2012

\begin{abstract}
Given a morphism from an affine semigroup to an arbitrary commutative monoid, it is shown that every fiber possesses an affine stratification: a partition into a finite disjoint union of translates of normal affine semigroups. The proof rests on mesoprimary decomposition of monoid congruences and a novel list of equivalent conditions characterizing the existence of an affine stratification. The motivating consequence of the main result is a special case of a conjecture due to Guo and the author on the existence of affine stratifications for (the set of winning positions of) any lattice game. The special case proved here assumes that the lattice game has finite misère quotient, in the sense of Plambeck and Siegel.
\end{abstract}

Keywords Affine semigroup · Lattice game - Mesoprimary decomposition · Misère quotient $\cdot$ Monoid

\section{Introduction}

Lattice games encode finite impartial combinatorial games - and winning strategies for them-in terms of lattice points in rational convex polyhedra [3]; see Sect. 4. The concept grew out of Plambeck's theory of misère quotients [10], as developed by Plambeck and Siegel [11] (see also Siegel's lecture notes [12], particularly Fig. 7 in Lecture 5 there). Their purpose was to provide data structures for recording and computing winning strategies of combinatorial games, such as octal games, under the misère play condition, where the last player to move loses. In that spirit, Guo and the author conjectured that the lattice points encoding the strategy of any lattice

The author had support from NSF grants DMS-0449102 = DMS-1014112 and DMS-1001437.

\footnotetext{
E. Miller $(\bowtie)$

Mathematics Department, Duke University, Durham, NC 27708, USA

e-mail: ezra@math.duke.edu

url: http://math.duke.edu/ ezra
} 
game have a particularly well-behaved presentation, called an affine stratification: a partition into a finite disjoint union of translates of affine semigroups [3, Conjecture 8.9]. The conjecture is as far from true as possible, in general, because lattice games support universal computation, as shown by Fink [2]. However, the most successful applications of misère quotients thus far have occurred when the quotient is finite, because of amenability to algorithmic computation. Bridging misère quotient theory and lattice games in the case of finite quotients is therefore one of the primary intents of this note, whose motivating result is the existence of affine stratifications for lattice games with finite misère quotients (Theorem 4.6).

The proof comes via a more general main result, of independent interest, on morphisms from affine semigroups to arbitrary commutative monoids: the fibers of such morphisms possess affine stratifications (Theorem 3.3). That result, in turn, follows from two additional results of independent interest. The first is a host of equivalent conditions characterizing the existence of an affine stratification (Theorem 2.7). The second is the existence of mesoprimary decomposition for congruences on finitely generated commutative monoids, a theory whose development occupies half of a full-length paper of its own [5]. One of the motivations for defining mesoprimary decomposition in the first place was its anticipated relevance to combinatorial game theory, via the results presented here and via potential further applications toward the existence of affine stratifications for the class of squarefree lattice games [3, Corrigendum] (which includes all octal games), where the affine stratification conjecture remains open.

Beyond mesoprimary decomposition and other more elementary theory of commutative monoids, the reasoning in this note involves elementary polyhedral geometry, including subdivisions and Minkowski sums, as well as combinatorial commutative algebra of finely graded modules over affine semigroup rings, particularly filtrations thereof.

\section{Affine stratifications}

The goal is to decompose certain sets of lattice points in polyhedra in particularly nice ways, following [3, Sect. 8], where this definition originates.

Definition 2.1 An affine stratification of a subset $\mathcal{W} \subseteq \mathbb{Z}^{d}$ is a finite partition

$$
\mathcal{W}=\biguplus_{i=1}^{r} W_{i}
$$

of $\mathcal{W}$ into a finite disjoint union of sets $W_{i}$, each of which is a finitely generated module for an affine semigroup $A_{i} \subseteq \mathbb{Z}^{d}$; that is, $W_{i}=F_{i}+A_{i}$, where $F_{i} \subseteq \mathbb{Z}^{d}$ is a finite set and $A_{i}$ is a finitely generated submonoid of the free abelian group $\mathbb{Z}^{d}$ of rank $d$.

For the coming sections, it will be helpful to specify, in Theorem 2.7, some alternative decompositions equivalent to affine stratifications. For that, we need four lemmas as stepping stones. In the first, a normal affine semigroup is the intersection of a rational polyhedral cone in $\mathbb{R}^{d}$ with a sublattice of $\mathbb{Z}^{d}$. 
Lemma 2.2 Every affine semigroup $A \subseteq \mathbb{Z}^{d}$ possesses an affine stratification in which each stratum is a translate $f_{i}+A_{i}$ of a normal affine semigroup $A_{i} \subseteq \mathbb{Z}^{d}$.

Proof Let $\bar{A}=\mathbb{R}_{+} A \cap \mathbb{Z} A$ denote the saturation of $A$ : the set of lattice points in the intersection of the real cone generated by $A$ with the group generated by $A$. Then $A$ contains a translate $\mathbf{a}+\bar{A}$ of its saturation by [9, Exercise 7.15]. Transferring this statement to the language of monoid algebras, the complex affine semigroup ring $\mathbb{C}[A]$ has a $\mathbb{C}[A]$-submodule $\mathbf{x}^{\mathbf{a}} \mathbb{C}[\bar{A}] \subseteq \mathbb{C}[A]$. The quotient $M=\mathbb{C}[A] / \mathbf{x}^{\mathbf{a}} \mathbb{C}[\bar{A}]$ is a finitely generated $\mathbb{Z}^{d}$-graded $\mathbb{C}[A]$-module. The module $M$ is therefore toric, in the sense of [6, Definition 4.5], by [6, Example 4.7]. This means that $M$ has a toric filtration $0=M_{0} \subset M_{1} \subset \cdots \subset M_{\ell-1} \subset M_{\ell}=M$, in which $M_{j} / M_{j-1}$ is, for each $j$, a $\mathbb{Z}^{d}$-graded translate $\mathbf{x}^{\mathbf{a}_{j}} \mathbb{C}\left[F_{j}\right]$ of the affine semigroup ring $\mathbb{C}\left[F_{j}\right]$ for some face $F_{j}$ of $A$ and some $\mathbf{a}_{j} \in \mathbb{Z}^{d}$. Transferring this statement back into the language of lattice points,

$$
A=(\mathbf{a}+\bar{A}) \uplus \biguplus_{j}\left(\mathbf{a}_{j}+F_{j}\right)
$$

is a disjoint union of a translated normal affine semigroup $\mathbf{a}+\bar{A}$ and a disjoint union of translates $\mathbf{a}_{j}+F_{j}$ of faces of $A$. But $\operatorname{dim} M<\operatorname{dim} \mathbb{C}[A]$, since $\operatorname{dim} \mathbf{x}^{\mathbf{a}} \mathbb{C}[\bar{A}]=$ $\operatorname{dim} \mathbb{C}[A]$, so each face $F_{j}$ that appears is a proper face of $A$. Therefore the proof is done by induction on $\operatorname{dim} \mathbb{C}[A]$, the case of dimension 0 being trivial, since then $A=\{0\}$.

In the next lemma, keep in mind that the polyhedra need not be bounded.

Lemma 2.3 Any finite union of (rational) convex polyhedra in $\mathbb{R}^{d}$ can be expressed as a disjoint union of finitely many sets, each of which is the relative interior of a (rational) convex polyhedron.

Proof The polyhedra in the given union $U$ are defined as intersections of finitely many halfspaces. The totality of all hyperplanes involved subdivide the ambient space into finitely many closed-but perhaps unbounded-polyhedral regions. This union of regions is a polyhedral complex (see [14, Sect. 5.1] for the definition) with finitely many faces, some of which may be unbounded. By construction, the relative interior of each face is either contained in $U$ or disjoint from $U$, proving the lemma.

The following will be used in the proofs of both Lemma 2.6 and Theorem 2.7.

Lemma 2.4 If $\Pi=P+C$ is a rational convex polyhedron in $\mathbb{R}^{d}$, expressed as the Minkowski sum of a polytope $P$ and a cone $C$ [14, Theorem 1.2], and $\Pi^{\circ}$ is its relative interior, then $\Pi \cap \mathbb{Z}^{d}$ and $\Pi^{\circ} \cap \mathbb{Z}^{d}$ are finitely generated modules for $A=C \cap \mathbb{Z}^{d}$.

Proof Suppose that $\Pi=\bigcap_{j}\left\{\mathbf{x} \in \mathbb{R}^{d} \mid \phi_{j}(\mathbf{x}) \geq c_{j}\right\}$ for rational linear functions $\phi_{j}$ and rational constants $c_{j}$. The case of $\Pi^{\circ}$ follows from that of $\Pi$ itself: the lattice points in $\Pi^{\circ}$ are the same as those in the closed polyhedron $\Pi_{\varepsilon}=\bigcap_{j}\left\{\mathbf{x} \in \mathbb{R}^{d} \mid\right.$ $\left.\phi_{j}(\mathbf{x}) \geq c_{j}+\varepsilon\right\}$ obtained from $\Pi$ by moving each of its bounding hyperplanes inward 
by a small rational distance, where $\varepsilon$ is less than any nonzero positive value of $\phi_{j}$ on $\mathbb{Z}^{d}$ for all $j$. (The rationality of $\phi_{j}$ guarantees that $\phi_{j}\left(\mathbb{Z}^{d}\right)$ is a discrete subset of the rational numbers $\mathbb{Q}$.)

Given the closed polyhedron $\Pi$, consider its homogenization [14, Sect. 1.5]: the closure $\bar{\Pi}$ of the cone over a copy of $\Pi=\Pi \times\{1\}$ placed at height 1 in $\mathbb{R}^{d+1}=$ $\mathbb{R}^{d} \times \mathbb{R}$. The intersection of $\bar{\Pi}$ with the first factor $\mathbb{R}^{d}=\mathbb{R}^{d} \times\{0\}$ is the cone $C$, and $\bar{\Pi} \cap \mathbb{Z}^{d}=A$ is a face of the affine semigroup $\bar{A}=\bar{\Pi} \cap \mathbb{Z}^{d+1}$. The intersection $\Pi \cap \mathbb{Z}^{d}$ is isomorphic, as a module over $A$, to the intersection $M=\bar{\Pi} \cap\left(\mathbb{Z}^{d} \times\{1\}\right)=$ $\bar{A} \cap\left(\mathbb{Z}^{d} \times\{1\}\right)$ with the copy of $\mathbb{Z}^{d}$ at height 1 .

To conclude that $M$ is finitely generated over $A$, it is enough (in fact, equivalent) to show that the vector space $\mathbb{C}\{M\}$ over the field $\mathbb{C}$ of complex numbers with basis $M$ is finitely generated as a module over the affine semigroup ring $\mathbb{C}[A]$. This follows because $\mathbb{C}\{M\}=\mathfrak{p}_{A} / \mathfrak{p}_{A}^{2}$ is finitely generated as a module over $\mathbb{C}[\bar{A}]$ and naturally annihilated by the kernel $\mathfrak{p}_{A}$ of the surjection $\mathbb{C}[\bar{A}] \rightarrow \mathbb{C}[A]$.

Remark 2.5 The $i$ th power $\mathfrak{p}_{A}^{i}$ of the prime ideal $\mathfrak{p}_{A}$ in the preceding proof is the ideal $\mathbb{C}[\bar{A}]_{>i}$ generated by all monomials whose exponent vectors in $\mathbb{Z}^{d} \times \mathbb{Z}$ have $\mathbb{Z}$-coordinate strictly greater than $i$. This ideal is an irreducible monomial ideal by $[7$, Eq. (1) and Lemma 2.2] or [9, Theorem 11.13], since the quotient $\mathbb{C}[\bar{A}] / \mathbb{C}[\bar{A}]_{>i}$ can be expressed as the $\bar{A}$-graded part of the indecomposable injective $\mathbb{C}\left\{i \mathbf{e}_{d+1}+A-\bar{A}\right\}$, where $\mathbf{e}_{d+1}=\mathbf{0} \times 1 \in \mathbb{Z}^{d} \times \mathbb{Z}$ is the last standard basis vector. In this guise, the preceding proof is inspired by (and based on) the proof of [1, Proposition 2.13].

Lemma 2.6 If $\mathcal{W} \subseteq \mathbb{Z}^{d}$ is a finite union of sets $W_{i}$, each a translate of a normal affine semigroup $A_{i}=\mathbb{R}_{+} A_{i} \cap L_{i}$ for some sublattice $L_{i} \subseteq \mathbb{Z}^{d}$, then $\mathcal{W}$ can be expressed as such a union in which $L_{i}=L$ for all $i$ is a fixed sublattice of finite index in $\mathbb{Z}^{d}$.

Proof Suppose that $A_{i}=\mathbb{R}_{+} A_{i} \cap L_{i}$ is given for all $i$. Taking a direct sum with a complementary sublattice, we may assume that $L_{i}$ has finite index in $\mathbb{Z}^{d}$ for all $i$. Now set $L=\bigcap_{i} L_{i}$. Then $A_{i}=\bigcup_{\lambda \in L_{i} / L} A_{i} \cap(\lambda+L)$ is a finite union of sets obtained by intersecting a coset of $L$ with $A_{i}$. Each such set $A_{i} \cap(\lambda+L)$ is a finitely generated module over $A_{i} \cap L$ by Lemma 2.4. The desired union is therefore achievable using translates of the normal affine semigroups $A_{i} \cap L$.

Theorem 2.7 The following are equivalent for a set $\mathcal{W} \subseteq \mathbb{Z}^{d}$ of lattice points.

1. $\mathcal{W}$ possesses an affine stratification.

2. $\mathcal{W}$ is a finite (not necessarily disjoint) union of sets $W_{i}$, each of which is a finitely generated module for an affine semigroup $A_{i} \subseteq \mathbb{Z}^{d}$.

3. $\mathcal{W}$ is a finite (not necessarily disjoint) union of sets $W_{i}$, each of which is a translate $f_{i}+A_{i}$ of an affine semigroup $A_{i} \subseteq \mathbb{Z}^{d}$.

4. $\mathcal{W}$ is a finite (not necessarily disjoint) union of sets $W_{i}$, each of which is a translate $f_{i}+A_{i}$ of a normal affine semigroup $A_{i} \subseteq \mathbb{Z}^{d}$.

5. $\mathcal{W}$ is a finite disjoint union of sets $W_{i}$, each of which is a translate $f_{i}+A_{i}$ of a normal affine semigroup $A_{i} \subset \mathbb{Z}^{d}$.

6. $\mathcal{W}$ is a finite disjoint union of sets $W_{i}$, each of which is a translate $f_{i}+A_{i}$ of a (not necessarily normal) affine semigroup $A_{i} \subset \mathbb{Z}^{d}$. 
Proof By definition it follows that $1 \Rightarrow 2 \Rightarrow 3$ and that $5 \Rightarrow 6 \Rightarrow 1$. It therefore remains only to show that $3 \Rightarrow 4 \Rightarrow 5$. The first of these implications is Lemma 2.2.

For the second, begin by choosing the union to satisfy the conclusion of Lemma 2.6. For each $\lambda \in \mathbb{Z}^{d} / L$, let $\mathcal{W}_{\lambda}=\mathcal{W} \cap(\lambda+L)$ be the intersection of $\mathcal{W}$ with the corresponding coset of $L$ in $\mathbb{Z}^{d}$. Then $\mathcal{W}_{\lambda}$ is the intersection of $\lambda+L$ with the union $U_{\lambda}$ of those polyhedra $f_{i}+\mathbb{R}_{+} A_{i}$ for which $f_{i} \in \lambda+L$. By Lemma 2.3, it suffices to show that if $W$ is the intersection of $L$ with the relative interior of a polyhedron, then $W$ possesses an affine stratification in which every stratum is a translate of a normal affine semigroup. Replacing $L$ with $\mathbb{Z}^{d}$, we may as well assume that $L=\mathbb{Z}^{d}$. Lemma 2.4 implies that $W$ is a finitely generated module over a normal affine semigroup $A$. Thus the vector space over $\mathbb{C}$ with basis $W$ constitutes a finitely generated $\mathbb{Z}^{d}$-graded submodule $M \subseteq \mathbb{C}\left[\mathbb{Z}^{d}\right]$ over the affine semigroup ring $\mathbb{C}[A]$. The result now follows by a simpler version of the argument in the proof of Lemma 2.2, using a toric filtration: in the present case, dimension is not an issue, and $M_{j} / M_{j-1}$ is already a $\mathbb{Z}^{d}$-graded translate of a normal affine semigroup ring, because every face of $A$ is normal.

Corollary 2.8 Fix a linear map $\varphi: \mathbb{Z}^{n} \rightarrow \mathbb{Z}^{d}$. If $\mathcal{W} \subseteq \mathbb{Z}^{n}$ possesses an affine stratification then so does $\varphi(\mathcal{W}) \subseteq \mathbb{Z}^{d}$.

Proof The image of any translate of an affine semigroup in $\mathbb{Z}^{n}$ is a translate of an affine semigroup in $\mathbb{Z}^{d}$, so use a stratification of $\mathcal{W}$ as in Theorem 2.7.6: nothing guarantees that the images of the strata are disjoint, but that is irrelevant by Theorem 2.7.3.

Corollary 2.9 If each of finitely many given subsets of $\mathbb{Z}^{d}$ possesses an affine stratification, then so does their union.

Proof Use the equivalence of (for example) Theorem 2.7.1 and Theorem 2.7.2.

Remark 2.10 The reason for choosing Definition 2.1 as the fundamental concept instead of the other conditions in Theorem 2.7 is that Definition 2.1 likely results in the most efficient data structure for algorithmic purposes; see [4].

\section{Fibers of affine presentations of commutative monoids}

This section serves as an elementary example of the theory of mesoprimary decomposition and as a bridge to combinatorial game theory in the presence of finite misère quotients considered in Sect. 4. The main observation in this section is that affine stratifications exist for fibers of affine presentations of arbitrary commutative monoids. The relevant statement in Theorem 3.3 requires no additional background, but its more detailed final claim invokes notions from mesoprimary decomposition [5]. The prerequisites for the statement are [5, Definitions 2.11 and 5.2]; the proofs-including the definition of universal group - also need [5], particularly Definitions 2.8, 3.10, 6.2, 6.5, and 8.1, Example 3.11, Corollary 6.6, and Theorem 8.3 there. 
Lemma 3.1 To specify a prime congruence [5, Definition 2.11] on an affine semigroup it is equivalent to pick a face $F$ and subgroup $L \subseteq \mathbb{Z} F$ of the universal group of $F$.

Proof The face $F$ is the set of elements of the ambient affine semigroup $A$ whose images are not nil [5, Definition 2.8]. The subgroup $L$ is the kernel of the group homomorphism obtained by localizing the monoid morphism $F \rightarrow A / \sim$ along $F$ [5, Definition 3.10], where $\sim$ is the prime congruence in question.

Definition 3.2 A lattice $L \subseteq \mathbb{Z} A$ is associated to a congruence $\sim$ on an affine semigroup $A$ if $\sim$ has an associated prime congruence [5, Definition 5.2] specified by $L \subseteq \mathbb{Z} F$ for some face $F$ as in Lemma 3.1, and in this case $F$ is an associated face of $\sim$.

Theorem 3.3 If $\varphi: A \rightarrow Q$ is a surjection of commutative monoids with $A$ an affine semigroup, then every fiber of $\varphi$ possesses an affine stratification. Moreover, the stratification can be chosen so that each of its affine semigroups is $L \cap A$ for some intersection $L$ of associated lattices of the congruence defining $\varphi$.

The proof, included after Proposition 3.6, requires some preliminary results on affine stratifications in simpler situations than Theorem 3.3.

Lemma 3.4 If $M$ is an ideal in an affine semigroup $A$, then $M$ possesses an affine stratification in which each stratum is a translate $f_{i}+A_{i}$ of a face $A_{i}$ of $A$.

Proof Use a toric filtration as in the proof of Lemma 2.2, where $M$ there is replaced by the $\mathbb{C}[A]$-module $\mathbb{C}\{M\}$ that has the ideal $M \subseteq A$ as a vector space basis over $\mathbb{C}$.

Example 1 Every ideal in $\mathbb{N}^{n}$ has a Stanley decomposition [13]: an expression as a finite disjoint union of translates of faces $\mathbb{N}^{J}$ of $\mathbb{N}^{n}$ for $J \subseteq\{1, \ldots, n\}$; see [8, Sect. 2].

Lemma 3.5 Fix a normal affine semigroup A. The intersection $(\mathbf{u}+L) \cap M$ of any coset of a lattice $L \subseteq \mathbb{Z} A$ with an ideal $M \subseteq A$ is a finitely generated module for $L \cap A$.

Proof The ideal $M$ is finitely generated, and the intersection $(\mathbf{u}+L) \cap(\mathbf{a}+A)$ is finitely generated as a module over $L \cap A$ for any $\mathbf{a} \in A$ by Lemma 2.4.

Proposition 3.6 If $L$ is the associated lattice of a mesoprimary congruence on an affine semigroup A, then every non-nil congruence class is a finite union of sets $(\mathbf{a}+L) \cap A$.

Proof Let $F$ be the associated face, and write $Q$ for the quotient of $A$ modulo the mesoprimary congruence. Since the localization morphism $Q \rightarrow Q_{F}$ along $F$ is injective, it suffices to show that every element of $Q_{F}$, each viewed as a subset of the localization $A_{F}=A+\mathbb{Z} F$, is a finite union of cosets of $L$. Under the action of $\mathbb{Z} F$ 
on $Q_{F}$, the stabilizer of every non-nil element $q \in Q_{F}$ is $L$; this is what semifreeness means in [5, Corollary 6.6]. Consequently, viewing $q$ as a subset of $A_{F}$, the intersection of $q$ with any single coset of $\mathbb{Z} F$ is a single coset of $L$. Hence the result follows from finiteness of the number of $F$-orbits [5, Corollary 6.6].

Proof of Theorem 3.3 Let $\sim$ be the congruence on $A$ induced by $\varphi$. The fiber over the nil of $Q$, if there is one, is an ideal of $A$, which has an affine stratification by Lemma 3.4. To treat the non-nil fibers, fix a mesoprimary decomposition of $\sim$ [5, Theorem 8.3]. Let $L_{i} \subseteq \mathbb{Z} F_{i}$ for $i=1, \ldots, r$ be the lattices associated to $\sim$ with corresponding associated faces $F_{i}$ of $A$. Write $\bar{Q}_{i}$ for the quotient of $A$ modulo the $i$ th mesoprimary congruence in the decomposition.

Every class $q \in Q$ is, as a subset of $A$, the intersection of the $r$ mesoprimary classes $\bar{q}_{i} \in \bar{Q}_{i}$ containing $q$, because $\sim$ is the common refinement of its mesoprimary components. Furthermore, as long as $q$ is not nil, at least one of the mesoprimary classes $\bar{q}_{i}$ is not nil. For any such non-nil class $\bar{q}_{i}$, Proposition 3.6 guarantees a finite set $U_{i}$ such that $\bar{q}_{i}=\bigcup_{\mathbf{u} \in U_{i}}\left(\mathbf{u}+L_{i}\right) \cap A$. Renumbering for convenience, assume that $\bar{q}_{i}$ is non-nil for $i \leq k$ and nil for $i>k$. Then $q^{\prime}:=\bar{q}_{1} \cap \cdots \cap \bar{q}_{k}$ is the intersection with $A$ of a finite union of cosets of the lattice $L_{1} \cap \cdots \cap L_{k}$, and $q^{\prime \prime}:=\bar{q}_{k+1} \cap \cdots \cap \bar{q}_{r}$ is an ideal of $A$. Since $q=q^{\prime} \cap q^{\prime \prime}$, the result follows from Lemma 3.5.

\section{Lattice games and misère quotients}

Fix a pointed rational convex polyhedron $\Pi \subseteq \mathbb{R}^{d}$ with recession cone $C$ of dimension $d$. The pointed hypothesis means that $\Pi=P+C$ for some polytope (i.e., bounded convex polyhedron) $P$. Write $\Lambda=\Pi \cap \mathbb{Z}^{d}$ for the set of integer points in $\Pi$. The following definitions summarize [3, Definition 2.3, Definition 2.9, and Lemma 3.5].

Definition 4.1 A finite subset $\Gamma \subset \mathbb{Z}^{d} \backslash\{\mathbf{0}\}$ is a rule set if

1. there exists a linear function on $\mathbb{R}^{d}$ that is positive on $\Gamma \cup C \backslash\{\mathbf{0}\}$; and

2. there is finite set $F \subset \Lambda$ such that every position $p \in \Lambda$ has a $\Gamma$-path in $\Lambda$ to $F$ : a sequence $p=p_{r}, \ldots, p_{0} \in \Lambda$ with $p_{0} \in F$ and $p_{k}-p_{k-1} \in \Gamma$ for $k=\{1, \ldots, r\}$.

For the next definition, it is important to observe that the rule set $\Gamma$ induces a partial order $\preceq$ on $\Lambda$ in which $p \preceq q$ if $q-p$ lies in the monoid $\mathbb{N} \Gamma$ generated by $\Gamma$ [3, Lemma 2.8]. An order ideal under this (or any) partial order $\preceq$ is a subset $S$ closed under going down: $p \preceq q$ and $q \in S \Rightarrow p \in S$.

Definition 4.2 Fix a rule set $\Gamma$.

- A game board $B$ is the complement in $\Lambda$ of a finite $\Gamma$-order ideal in $\Lambda$ called the set of defeated positions.

- A lattice game $G=(\Gamma, B)$ is defined by a rule set $\Gamma$ and a game board $B$.

- The $P$-positions of $G$ form a subset $\mathcal{P} \subseteq B$ such that $(\mathcal{P}+\Gamma) \cap B=B \backslash \mathcal{P}$. 
- An affine stratification of $G$ is an affine stratification of its set of $P$-positions.

The $P$-positions of $G$ are uniquely determined by the rule set and game board [3, Theorem 4.6].

Following Plambeck and Siegel $[10,11]$, every lattice game possesses a unique quotient that optimally collapses $\Lambda$ while faithfully recording the interaction of the $P$-positions with its additive structure.

Definition 4.3 Two positions $p, q \in B$ are indistinguishable, written $p \sim q$, if

$$
(p+C) \cap \mathcal{P}=p-q+(q+C) \cap \mathcal{P} .
$$

In other words, $p+r \in \mathcal{P} \Leftrightarrow q+r \in \mathcal{P}$ for all $r$ in the recession cone $C$ of $B$. The misère quotient of the lattice game with winning positions $\mathcal{P}$ is the quotient $\Lambda / \sim$ of the polyhedral set $\Lambda$ modulo indistinguishability.

Geometrically, indistinguishability means that the $P$-positions in the cone above $p$ are the same as those above $q$, up to translation by $p-q$. It is elementary to verify that indistinguishability is an equivalence relation, and that it is additive, in the sense that $p \sim q \Rightarrow p+r \sim q+r$ for all $r \in C \cap \mathbb{Z}^{d}$. Thus, when $B=\Lambda=C \cap \mathbb{Z}^{d}$ is a monoid, indistinguishability is a congruence, so the quotient of $B$ modulo indistinguishability is again a monoid.

Lemma 4.4 Every fiber of the projection $\Lambda \rightarrow \Lambda / \sim$ either consists of $P$-positions or has empty intersection with $\mathcal{P}$.

Proof If $p \sim q$, then by definition either $p$ and $q$ are both $P$-positions or neither is.

Corollary 4.5 Fix a lattice game $G=(\Gamma, B)$ played on a cone, meaning that the game board is the complement of the defeated positions in a normal affine semigroup $\Lambda$. If the misère quotient $\Lambda / \sim$ is finite, then $G$ admits an affine stratification.

Proof The set $\mathcal{P}$ of $P$-positions is a union of fibers of the projection. If the quotient is finite, then the union is finite. Now apply Theorem 3.3.

Although it is useful to record Corollary 4.5, which treats the case of finite misère quotient monoids, where the game is played on a cone, the extension to arbitrary finite misère quotients requires little additional work.

Theorem 4.6 Fix a lattice game $G=(\Gamma, B)$ played on a polyhedral set $\Lambda=\Pi \cap \mathbb{Z}^{d}$. If the misère quotient $\Lambda / \sim$ is finite, then $G$ admits an affine stratification.

Proof The set $\mathcal{P}$ of $P$-positions is a union of fibers of the projection $\Lambda \rightarrow \Lambda / \sim$. Since the quotient is finite, the union is finite. Therefore it suffices to show that every fiber $\Phi \subseteq \Lambda$ of the projection possesses an affine stratification.

The pointed hypothesis on $\Pi$ implies that $\Lambda=F+A$, where $F \subseteq \mathbb{Z}^{d}$ is finite and $A=C \cap \mathbb{Z}^{d}$ is a normal affine semigroup. The fiber $\Phi$ is a finite union $\Phi=$ 
$\bigcup_{f \in F} \Phi \cap(f+A)$. By Corollary 2.9, it therefore suffices to show that for each lattice point $f \in F$, every fiber of the map $f+A \rightarrow \Lambda / \sim$ possesses an affine stratification. But the composite map $A \rightarrow f+A \rightarrow \Lambda / \sim$ induces a congruence on $A$ whose classes are the fibers, to which Theorem 3.3 applies.

\section{References}

1. Dickenstein, A., Matusevich, L.F., Miller, E.: Combinatorics of binomial primary decomposition. Math. Z. 264(4), 745-763 (2010). arXiv:0803.3846 [math.AC]

2. Fink, A.: Lattice games without rational strategies. J. Comb. Theory, Ser. A 119, 450-459 (2012). arXiv:1106.1883 [math.CO]

3. Guo, A., Miller, E.: Lattice point methods for combinatorial games. Adv. Appl. Math. 46, 363378 (2011). arXiv:0908.3473 [math.CO] [Corrigendum: ibid. 48 (2012), 269-271. arXiv:1105.5420 [math.CO]]

4. Guo, A., Miller, E.: Algorithms for lattice games. Int. J. Game Theory (2012). doi:10.1007/ s00182-012-0319-9. arXiv:1105.5413 [math.AC]

5. Kahle, T., Miller, E.: Decompositions of commutative monoid congruences and binomial ideals. Preprint, 64 pages. arXiv:1107.4699 [math.AC]

6. Matusevich, L.F., Miller, E., Walther, U.: Homological methods for hypergeometric families. J. Am. Math. Soc. 18(4), 919-941 (2005). arXiv:math.AG/0406383

7. Miller, E.: Cohen-Macaulay quotients of normal semigroup rings via irreducible resolutions. Math. Res. Lett. 9(1), 117-128 (2002). arXiv:math.AC/0110096

8. Miller, E.: Topological Cohen-Macaulay criteria for monomial ideals. In: Combinatorial Aspects of Commutative Algebra. Contemp. Math., vol. 502, pp. 137-155. Am. Math. Soc., Providence (2009). arXiv:0809.1458 [math.AC]

9. Miller, E., Sturmfels, B.: Combinatorial Commutative Algebra. Graduate Texts in Mathematics, vol. 227. Springer, New York (2005)

10. Plambeck, T.E.: Taming the wild in impartial combinatorial games. Integers 5, G5 (2005) 36 pp. (electronic) arXiv:math.CO/0501315

11. Plambeck, T.E., Siegel, A.N.: Misère quotients for impartial games. J. Comb. Theory, Ser. A 115, 593-622 (2008). arXiv:math.CO/0609825

12. Siegel, A.N.: Misère games and misère quotients. Preprint. arXiv:math.CO/0612616 (2006)

13. Stanley, R.P.: Linear Diophantine equations and local cohomology. Invent. Math. 68, 175-193 (1982)

14. Ziegler, G.M.: Lectures on Polytopes. Graduate Texts in Mathematics, vol. 152. Springer, New York (1995) 